\title{
SINGULAR PERTURBATIONS AND NONSTANDARD ANALYSIS ${ }^{1}$ \\ BY
}

\author{
S. ALBEVERIO, J. E. FENSTAD AND R. HØEGH-KROHN
}

\begin{abstract}
We study by methods of nonstandard analysis second order differential operators with zero order coefficients which are too singular to be defined by standard functions. In particular we study perturbations of the Laplacian in $R^{3}$ given by potentials of the form $\lambda \Sigma_{j} \delta\left(x-x_{j}\right)$. We also study Sturm-Liouville problems with zero order coefficients given by measures and prove that they satisfy the same oscillation theorems as the regular Sturm-Liouville problems.
\end{abstract}

1. Introduction. Nonstandard analysis, a subject initiated by A. Robinson (see [1]), is by now a large body of knowledge, with applications in different fields, see e.g., the recent books [2], [3] and references therein. Parts of analysis where nonstandard analysis should be very useful are those involving singular perturbations, like the ones encountered in certain physical problems. For some work in this direction see e.g. [4] and references therein. In fact there are problems which find their natural formulation only in nonstandard analysis. In this paper we examine some examples of such problems. It is clear from the treatment however that the methods are suitable for handling much more general singular perturbation problems.

Let us in general terms try to give grounds for this belief by pointing to a similarity between nonstandard methods and applications of distribution theory. In both cases we have a mathematical structure $E$, in both cases we introduce "ideal" elements, in nonstandard analysis an extension ${ }^{*} E$, in distribution theory a completion $E^{\prime}$. In many cases it does not matter whether we work in the extension ${ }^{*} E$ or in $E^{\prime}$. But ${ }^{*} E$ has an extra algebraic and combinatorial richness which is lost in the distribution theoretic framework. Thus the methodological theme of our paper is that sometimes (but not necessarily always) this extra richness can be put to good use.

We can distinguish two parts in our paper. In the first one, consisting of $\S \S 2$ and 3 we examine perturbations of the Laplacian in $R^{3}$ by " $\delta$-functions". The second part, consisting of $\S 4$, handle the Sturm-Liouville theory with a

Received by the editors April 17, 1978 and, in revised form, July 12, 1978.

AMS (MOS) subject classifications (1970). Primary 26A98, 35B25, 35B05, 35J10, 81A09.

Key words and phrases. Singular perturbations, Schrödinger operators, Sturm-Liouville problems, nonstandard analysis.

'Work supported in part by the Norwegian Research Council for Science and the Humanities. 
zero order coefficient which is a measure. Let us now give some more details on the problems and the results of the different sections.

In $\$ 2$ we treat by nonstandard analysis a problem which has been formulated and discussed previously in terms of standard analysis by F. A. Berezin and L. D. Faddeev [5]. It arises in the quantum mechanical description of two particles interacting by a potential of the form " $\lambda \delta$ ", where $\delta$ is the delta function in $R^{3}$ and $\lambda$ is a constant. Recently a solution in terms of Dirichlet forms was given [6] as follows. To the formal operator $-\Delta+\lambda \delta$ there corresponds, for certain values of $\lambda$, a one-parameter family of selfadjoint operators $H_{\alpha}$ in $L^{2}\left(R^{3}\right)$. These can be characterized as the selfadjoint operators associated with the Dirichlet forms

$$
\left(H_{\alpha}^{1 / 2} f, H_{\alpha}^{1 / 2} f\right)=\int(\operatorname{grad} f)^{2} d \mu_{\alpha},
$$

where $d \mu_{\alpha}(x)=(\alpha / 2 \pi)\left(e^{-2 \alpha|x|} /|x|^{2}\right) d x, x \in \mathbf{R}^{3}$.

The construction of $H_{\alpha}$ that Berezin and Faddeev gave was in terms of characteristic functions in the Fourier transform space. C. Friedman [7] (see also [8]) has further discussed this problem and the corresponding problems in $\mathbf{R}^{d}$ of perturbations of the Laplacian by sequences of potentials with shrinking supports. In particular he has proved resolvent convergences of $-\Delta-(3 / 4 \pi) \lambda n^{2} \chi_{1 / n}$ as $n \rightarrow \infty$, where $\chi_{1 / n}$ is the characteristic function of the sphere of radius $1 / n$. The limit is $H_{\alpha}$ with $\alpha=0$, if $3 \lambda / \pi^{3}$ is an odd integer, and it is $-\Delta$ if $3 \lambda / \pi^{3}$ is not an odd integer. Friedman's results have been shown by Nelson, as an illustration to his paper on nonstandard analysis [9], to follow from the nonstandard perturbation problem $-\Delta-$ $\left(3 \lambda / 4 \pi \varepsilon^{2}\right) \chi_{\varepsilon}$, where $\varepsilon$ is an infinitesimal (in some nonstandard extension ${ }^{*} \mathbf{R}$ of R: see e.g. [2] for definitions concerning nonstandard analysis).

In $\$ 2$ of the present paper we bridge the gap between Nelson's nonstandard result and Berezin-Faddeev's standard one by a more refined choice of the nonstandard perturbation, in fact by taking $\lambda_{e}(\alpha) \chi_{e}, \lambda_{e}(\alpha)=[-(k$ $\left.+\frac{1}{2}\right)^{2} \pi^{2} / \varepsilon^{2}+2 \alpha / \varepsilon+\beta$, with $\alpha$ and $\beta$ real standard and $k$ an integer. We show that $H_{\alpha}=-\Delta+\lambda_{\varepsilon}(\alpha) \chi_{\varepsilon}$ is near standard and its standard part $H_{\alpha}$ is independent of $k, \beta$ and, as $\alpha$ runs through $\mathbf{R}, H_{\alpha}$ runs through all the selfadjoint extensions of the restriction of $-\Delta$ to $C_{0}^{\infty}\left(\mathbf{R}^{3}-\{0\}\right)$, i.e. $H_{\alpha}$ coincides with the above operators defined by Dirichlet forms. The method used in the proof is a refinement of Nelson's one and an extension to three dimensions of methods used before by Kelemen and Robinson [4] for one-dimensional quantum mechanical problems.

In $\$ 3$ we give the perturbation theory of the more general problems of the type $-\Delta-\lambda \sum_{i=1}^{n} \delta\left(x-x_{i}\right)$, where $x_{i}$ are fixed points in $\mathbf{R}^{3}$. We make precise the problem in terms of nonstandard analysis, as the problem of 
studying the selfadjoint operator $H$ associated with the closed form $-(f, \Delta f)$ $-\lambda \sum_{j=1}^{n}\left|\left(f, \delta_{\omega}\left(\cdot-x_{j}\right)\right)\right|^{2}$ on the nonstandard Hilbert space ${ }^{*} L_{2}\left(R^{3}\right)$, where $\delta_{\omega}$ is the Fourier transform of the characteristic function $\chi_{\omega}$ of the nonstandard ball $|x| \leqslant \omega$, with $\omega$ infinite. We show that for $\lambda=\lambda_{\omega}(\alpha)$,

$$
\lambda_{\omega}(\alpha)^{-1}=\int_{|p|<\omega} \frac{d p}{p^{2}+m^{2}}+\alpha, \quad \alpha \in \mathbf{R},
$$

we have that $\tilde{H}=\tilde{H}_{\alpha}$ is near standard, with $H=$ st $\tilde{H}$, and we give an explicit expression for $\left(\tilde{H}_{\alpha}+m^{2}\right)^{-1}, m>0, m \in \mathbf{R}$, by utilizing the Neumann series for the corresponding problem with a standard finite $\omega$, together with the transfer principle.

In $\S 4$ we take up the study of the Sturm-Liouville Dirichlet problem

$$
A u=-\left(p u^{\prime}\right)^{\prime}+\mu u
$$

on $L_{2}([a, b])$, with $\mu$ a positive measure. By associating to this problem the corresponding nonstandard Sturm-Liouville problem given by the second order nonstandard differential operator

$$
\tilde{A} \tilde{u}=-\left({ }^{*} p \tilde{u}^{\prime}\right)^{\prime}+q_{\mu} \tilde{u}
$$

on ${ }^{*} L_{2}([a, b])$, where $q_{\mu}$ is a smooth nonstandard version of the standard measure $\mu$, we are able to give a sense to the problem (1.1). We show that the standard results proven for the regular Sturm-Liouville problem hold also in our more singular situation. In particular we show that the selfadjoint operator associated with (1.1) has discrete spectrum consisting of simple eigenvalues $0<\lambda_{0}<\lambda_{1}<\cdots<\lambda_{n}<\cdots$, such that the corresponding eigenfunctions $u_{n}$ have exactly $n+2$ zeros, at which they change sign, and the zeros of $u_{n+1}$ separate those of $u_{n}$. These results follow using that the corresponding nonstandard differential operator $\tilde{A}$ has such properties.

For the terminology of nonstandard analysis we refer to [2].

2. Perturbations of the Laplacian by a potential with infinitesimal support. On $L_{2}\left(\mathbf{R}^{+}, d r\right), d r$ is the Lebesgue measure, consider the selfadjoint operator

$$
A=-d^{2} / d r^{2}+\lambda \chi_{\varepsilon}(r)
$$

with Dirichlet boundary conditions at $r=0$, where $\chi_{\varepsilon}$ is the characteristic function for the interval $[0, \varepsilon]$ for some $\varepsilon>0$. Let $v_{i}, i=1,2$, be solutions of the corresponding equation

$$
-u^{\prime \prime}+\lambda \chi_{\varepsilon} u-z u=0
$$

on $\mathbf{R}^{+}$with $z \in \mathbf{C}$, such that $z \notin \mathbf{R}^{+}$and $v_{i}(0)=0$ and $v_{2}(r) \rightarrow 0$ as $r \rightarrow \infty$. By the Sturm-Liouville theory we then have that

$$
K=v_{1} v_{2}^{\prime}-v_{1}^{\prime} v_{2}
$$

is independent of $r$ and if $K \neq 0$ then $z$ is not an eigenvalue of $A$, thus $z-A$ 
has a bounded inverse on $L_{2}\left(\mathbf{R}^{+}\right)$

$$
G_{z}=(z-A)^{-1},
$$

where the kernel of $G_{z}$ is given by

$$
G_{z}(x, y)=\frac{1}{K} \begin{cases}v_{1}(x) v_{2}(y), & x<y, \\ v_{1}(y) v_{2}(x), & y<x .\end{cases}
$$

From (2.2) we have, with $\operatorname{Re} \sqrt{z} \geqslant 0$, that

$$
v_{1}(r)= \begin{cases}\sin (\sqrt{z-\lambda} r), & 0 \leqslant r \leqslant \varepsilon, \\ a e^{\sqrt{2} r}+b e^{-\sqrt{2} r}, & \varepsilon \leqslant r,\end{cases}
$$

and

$$
v_{2}(r)= \begin{cases}c e^{\sqrt{\lambda-z} r}+d e^{-\sqrt{\lambda-z} r}, & 0<r<\varepsilon, \\ e^{-\sqrt{z} r}, & \varepsilon<r .\end{cases}
$$

From the continuity of $v_{i}(r)$ and $v_{i}^{\prime}(r)$ at $r=\varepsilon$ we get

$$
\begin{aligned}
& a=\frac{1}{2} e^{-\sqrt{z} \varepsilon}\left(\sin \sqrt{z-\lambda} \varepsilon+\sqrt{\frac{z-\lambda}{z}} \cos \sqrt{z-\lambda} \varepsilon\right), \\
& b=\frac{1}{2} e^{\sqrt{z} \varepsilon}\left(\sin \sqrt{z-\lambda} \varepsilon-\sqrt{\frac{z-\lambda}{z}} \cos \sqrt{z-\lambda} \varepsilon\right) .
\end{aligned}
$$

This gives

$$
K=-2 a \sqrt{z}
$$

We shall now assume that $a \neq 0$ which of course always is the case if $\operatorname{Im} z \neq 0$. In this case $G_{z}=(z-A)^{-1}$ is bounded.

Let us now introduce the hyper reals ${ }^{*} \mathbf{R}$, a nonstandard extension of the real numbers $\mathbf{R}$, and let accordingly ${ }^{*} L_{2}\left(\mathbf{R}^{+}\right)$be the corresponding nonstandard extension of the Hilbert space $L_{2}\left(\mathbf{R}^{+}\right)$. For any positive $\lambda \in{ }^{*} \mathbf{R}$ we then have a unique selfadjoint operator ${ }^{*} A$ given by $(2.1)$ on ${ }^{*} L_{2}\left(\mathbf{R}^{+}\right)$, where $\chi_{\varepsilon}$ now is the characteristic function for the interval $[0, \varepsilon] \subset * \mathbf{R}$. By the transfer principle all the calculations and formulas above still hold, so for any $\lambda \in{ }^{*} \mathbf{R}$ we still have that ${ }^{*} A$ is selfadjoint and if $\operatorname{Im} z \neq 0$ then ${ }^{*} G_{z}=(z$ $\left.{ }^{*} A\right)^{-1}$ is a bounded operator on ${ }^{*} L_{2}\left(\mathbf{R}^{+}\right)$. For $\varepsilon$ infinitesimal we are interested in the case where ${ }^{*} G_{z}$ is near standard, i.e. for any $f \in{ }^{*} L_{2}\left(\mathbf{R}^{+}\right)$ which is near standard we have that ${ }^{*} G_{z} f$ is near standard. Because in this case st $f \rightarrow$ st ${ }^{*} G_{2} f$ (st being the operation of taking the standard part) defines an everywhere defined hence bounded linear operator on $L_{2}\left(\mathbf{R}^{+}\right)$.

So take now $\varepsilon \in{ }^{*} \mathbf{R}$ to be positive and infinitesimal, i.e. near zero. Then ${ }^{*} G_{z}$ will be near standard if its kernel ${ }^{*} G_{z}(x, y)$ is a near standard function. 
From (2.4) we see that this happens if the constants $a$ and $b$ are near standard and $a$ is not infinitesimal.

If $\lambda$ is finite, i.e. near standard, and $\varepsilon$ is infinitesimal it follows easily from (2.7) that st $^{*} G_{z}(x, y)$ is nothing but the resolvent kernel of the operator $A_{0}=-d^{2} / d r^{2}$ with Dirichlet boundary conditions.

If on the other hand $\lambda$ is infinite, i.e. not near standard, then to have $a$ and $b$ near standard we choose $\lambda$ such that $\sin \sqrt{z-\lambda} \varepsilon$ and $\sqrt{z-\lambda} \cos \sqrt{z-\lambda} \varepsilon$ both are near standard. But then $\cos \sqrt{z-\lambda} \varepsilon$ must be infinitesimal, i.e.

$$
\sqrt{z-\lambda} \varepsilon=\left(k+\frac{1}{2}\right) \pi+\eta
$$

where $k \in{ }^{*} N$ and $\eta$ is infinitesimal and so small that

$$
\tilde{\alpha}=\sqrt{z-\lambda} \cos \sqrt{z-\lambda} \varepsilon(-1)^{k}
$$

is near standard. It is easily seen that $\tilde{\alpha}$ is near standard iff $\sqrt{z-\lambda} \sin \eta$ is near standard and with $\lambda$ given in (2.9) this is equivalent with the quotient $\eta / \varepsilon$ being near standard. From (2.9) we have

$$
\lambda=-\left(k+\frac{1}{2}\right)^{2} \frac{\pi^{2}}{\varepsilon^{2}}-2 \frac{\pi}{\varepsilon}\left(k+\frac{1}{2}\right) \frac{\eta}{\varepsilon}+z-\left(\frac{\eta}{\varepsilon}\right)^{2} .
$$

From (2.10) we get with $\gamma=\eta / \varepsilon$ and $\gamma_{0}=$ st $\gamma$

$$
\text { st } \begin{aligned}
\tilde{\alpha} & =\left(k+\frac{1}{2}\right) \pi \text { st } \frac{\gamma}{\eta} \cos \left(\left(k+\frac{1}{2}\right) \pi+\eta\right)(-1)^{k} \\
& =(-1)^{k+1}\left(k+\frac{1}{2}\right) \pi \gamma_{0} \sin \left(k+\frac{1}{2}\right) \pi=-\left(k+\frac{1}{2}\right) \gamma_{0} \pi .
\end{aligned}
$$

So if $\tilde{\alpha}$ is near standard then we must take $k \in N$, on the other hand if we do take $k \in N$ and $\eta=\gamma \varepsilon$ with $\gamma \in \mathbf{R}$ then

$$
\alpha=\text { st } \tilde{\alpha}=-\left(k+\frac{1}{2}\right) \gamma \pi \text {. }
$$

Moreover then $\lambda$ takes the form

$$
\lambda=-\left(k+\frac{1}{2}\right)^{2} \frac{\pi^{2}}{\varepsilon^{2}}+\frac{2}{\varepsilon} \alpha+z-\gamma^{2}
$$

where $\alpha$ and $\gamma$ are in $\mathbf{R}$. We also get with this choice of $\lambda$ that

$$
\text { st } a=(-1)^{k} \frac{1}{2}\left(1+\frac{\alpha}{\sqrt{z}}\right), \quad \text { st } b=(-1)^{k} \frac{1}{2}\left(1-\frac{\alpha}{\sqrt{z}}\right)
$$

so that

$$
\text { st } \frac{a+b}{\sqrt{z}(a-b)}=1 / \alpha .
$$

From (2.16) however we see that

$$
\text { st } v_{1}(\varepsilon) / v_{1}^{\prime}(\varepsilon)=1 / \alpha,
$$


so that $u_{1}=$ st $v_{1}$ satisfies the equation

$$
-u_{1}^{\prime \prime}-z u=0
$$

and the boundary condition

$$
\alpha u_{1}(0)=u_{1}^{\prime}(0)
$$

Moreover since $u_{2}=$ st $v_{2}$ satisfies (2.18) and tends to zero at infinity we have that

$$
\text { st } G_{z}(x, y)=\frac{1}{\text { st } K} \begin{cases}u_{1}(x) u_{2}(y), & x \leqslant y, \\ u_{1}(y) u_{2}(x), & x \geqslant y,\end{cases}
$$

is the kernel of the resolvent for the selfadjoint operator $A_{\alpha}$ given as $-d^{2} / d r^{2}$ with boundary condition (2.19) on $L_{2}\left(\mathbf{R}^{+}\right)$.

It is easy to see that st $G_{z}(x, y)$ does not change if we add a near standard number to $\lambda$. Hence we may take $\lambda$ to be equal to

$$
\lambda=-\left(k+\frac{1}{2}\right)^{2} \frac{\pi^{2}}{\varepsilon^{2}}+\frac{2}{\varepsilon} \alpha .
$$

Moreover we have seen that st $G_{z}(x, y)$ does not depend on $k$ hence we may take $k=0$. We have thus the following theorem.

THEOREM 2.1. Consider the selfadjoint operator

$$
A_{\alpha}=-d^{2} / d r^{2}+\lambda_{\varepsilon}(\alpha) \chi_{\varepsilon}
$$

with Dirichlet boundary conditions on the nonstandard Hilbert space ${ }^{*} L_{2}\left(\mathbf{R}^{+}, d r\right)$, where

$$
\lambda_{\varepsilon}(\alpha)=-\left(k+\frac{1}{2}\right)^{2} \frac{\pi^{2}}{\varepsilon^{2}}+\frac{2}{\varepsilon} \alpha+\beta
$$

with $\alpha$ and $\beta$ in $\mathbf{R}, \varepsilon \in{ }^{*} \mathbf{R}, \varepsilon$ positive and infinitesimal, $k \in N$, and $\chi_{\boldsymbol{\varepsilon}}$ the characteristic function of the nonstandard interval $[0, \varepsilon] \subset{ }^{*} \mathbf{R}$. Then $A_{\alpha}$ is near standard in the sense that its resolvent $\left(A_{\alpha}+z\right)^{-1}$ is near standard. Moreover its standard part is the selfadjoint operator $-d^{2} / d r^{2}$ with boundary conditions $\alpha u(0)=u^{\prime}(0)$, in the sense that $\operatorname{st}\left(A_{\alpha}+z\right)^{-1}$ is the resolvent of this operator in the standard Hilbert space $L_{2}\left(\mathbf{R}^{+}, d r\right)$. Especially the standard part of $A_{\alpha}$ does not depend on $k \in N$ or $\beta \in \mathbf{R}$.

If $\dot{A}$ is the restriction of $-d^{2} / d r^{2}$ to the $C_{0}^{\infty}\left(\mathbf{R}^{+}\right)$-functions, then $\dot{A}$ is a symmetric operator on $L_{2}\left(\mathbf{R}^{+}, d r\right)$. It is well known that the selfadjoint extensions of $\dot{A}$ are $-d^{2} / d r^{2}$ with boundary conditions $\alpha u(0)=u^{\prime}(0)$. Hence we have the following corollary.

COROllaRy 2.2. Any selfadjoint extension of the restriction of $-d^{2} / d r^{2}$ to $C_{0}^{\infty}\left(\mathbf{R}^{+}\right)$is of the form of the standard part of $-d^{2} / d r^{2}+\lambda \chi_{\ell}$ on ${ }^{*} L_{2}\left(\mathbf{R}^{+}, d r\right)$ with any fixed infinitesimal $\varepsilon$ and some appropriately chosen $\lambda$. 
Now let $\Delta=\sum_{i=1}^{3} \partial^{2} / \partial x_{i}^{2}$ be the Laplacian as a selfadjoint operator in the standard Hilbert space $L_{2}\left(\mathbf{R}^{3}\right)$. We are interested in selfadjoint extensions of the restriction $₫$ of $\Delta$ to $C_{0}^{\infty}\left(\mathbf{R}^{3}-\{0\}\right)$. Now in polar coordinates $\Delta$ takes the form

$$
\Delta=\frac{\partial^{2}}{\partial r^{2}}+\frac{2}{r} \frac{\partial}{\partial r}+\frac{1}{r^{2}} B
$$

where $B$ is the Laplace-Beltrami operator on $L_{2}\left(S^{2}\right) . B$ has discrete spectrum with the eigenvalues $-l(l+1), l \in \mathbf{Z}^{+}$, with finite multiplicity. Hence to study $\Delta$ it is enough to study the operators

$$
\Delta_{l}=\frac{d^{2}}{d r^{2}}+\frac{2}{r} \frac{d}{d r}-\frac{l(l+1)}{r^{2}}
$$

on $L_{2}\left(\mathbf{R}^{+}, r^{2} d r\right)$, and to study the selfadjoint extensions of $₫$ is equivalent to study the selfadjoint extensions of the restriction $\Delta_{l}$ of $\Delta_{l}$ to $C_{0}^{\infty}\left(\mathbf{R}^{+}\right)$. Now (2.24) is unitarily equivalent to

$$
d^{2} / d r^{2}-l(l+1) / r^{2}
$$

on $L_{2}\left(\mathbf{R}^{+}, d r\right)$, and the unitary equivalence is given by $f(r) \leftrightarrow r f(r)$, so in particular $C_{0}^{\infty}\left(\mathbf{R}^{+}\right)$is carried onto itself by this unitary equivalence. Thus we shall study the selfadjoint extensions of the restriction of $(2.25)$ to $C_{0}^{\infty}\left(\mathbf{R}^{+}\right)$. It is well known, see for instance [10], that $(2.25)$ is essentially selfadjoint for $l \geqslant 1$. We have therefore only to consider the selfadjoint extensions of $-d^{2} / d r^{2}$ on $C_{0}^{\infty}\left(\mathbf{R}^{+}\right)$. These however are characterized in Corollary 2.2.

Let $\Delta$ also denote the selfadjoint Laplacian in the nonstandard Hilbert space ${ }^{*} L_{2}\left(\mathbf{R}^{3}\right)$. Let us consider the selfadjoint perturbation

$$
H_{\lambda}=-\Delta+\lambda \chi_{\varepsilon}(|x|)
$$

where $\chi_{\varepsilon}=\chi_{\varepsilon}(|x|)$ is the characteristic function for the nonstandard ball $|x| \leqslant \varepsilon . \varepsilon$ will be a fixed infinitesimal. We want to know for which values of $\lambda$ the operator $H_{\lambda}$ is near standard in the sense that its resolvent is near standard. If we split the Hilbert space ${ }^{*} \mathcal{H}={ }^{*} L_{2}\left(\mathbf{R}^{3}\right)$ into its rotationally symmetric part $* \mathcal{H}_{s}$ and its orthogonal complement $* \mathcal{H}_{s}^{\perp}$,

$$
* \mathscr{H}=* \mathcal{H}_{s} \oplus * \mathcal{H}_{s}^{\perp}
$$

we get from the fact established above, namely that the restriction of $\Delta$ to $C_{0}^{\infty}\left(\mathbf{R}^{3}\right) \cap \mathcal{H}_{s}^{\perp}$ is essentially selfadjoint, that the restriction of $H_{\lambda}$ to $* \mathcal{H}_{s}^{\perp}$ is near standard and its standard part is simply the same as that of $\Delta$. Hence we may limit our investigation to the restriction of $H_{\lambda}$ to $* \mathcal{H}_{s}$. But this restriction is unitarily equivalent, by the equivalence $f(r) \leftrightarrow r f(r)$, to the operator $(2.1)$ in ${ }^{*} L_{2}\left(\mathbf{R}^{+}, d r\right)$. From the results above and Theorem 2.1 we therefore have 
THEOREM 2.3. Consider the selfadjoint operator

$$
H_{\alpha}=-\Delta+\lambda_{\varepsilon}(\alpha) \chi_{\varepsilon}
$$

on the nonstandard Hilbert space ${ }^{*} L_{2}\left(\mathbf{R}^{3}\right)$, where

$$
\lambda_{\varepsilon}(\alpha)=-\left(k+\frac{1}{2}\right)^{2} \frac{\pi^{2}}{\varepsilon^{2}}+\frac{2}{\varepsilon} \alpha+\beta
$$

with $\alpha$ and $\beta$ in $\mathbf{R}, k \in N$ and $\varepsilon$ a fixed positive infinitesimal in ${ }^{*} \mathbf{R}$ and $\chi_{\varepsilon}$ is the characteristic function for the nonstandard ball $|x| \leqslant \varepsilon$ in ${ }^{*} \mathbf{R}^{3}$. Then $H_{\alpha}$ is near standard in the sense that its resolvent $\left(H_{\alpha}+z\right)^{-1}$ is near standard. Moreover its standard part does not depend on $k \in N$ or $\beta \in \mathbf{R}$ but is different for different $\alpha$. As $\alpha$ runs through $\mathbf{R}$, st $H_{\alpha}$ (the standard operator with resolvent equal to $\left.\operatorname{st}\left(H_{\alpha}+z\right)^{-1}\right)$ runs through all the selfadjoint extensions of the restriction of $\Delta$ to $C_{0}^{\infty}\left(\mathbf{R}^{3}-\{0\}\right)$.

3. Perturbations of the Laplacian by a potential with support in a finite set. Consider a perturbation of the Laplacian of the form

$$
-\Delta-\lambda \sum_{i=1}^{n} \delta\left(x-x_{i}\right)
$$

where $\Delta$ is the selfadjoint Laplacian in $L_{2}\left(\mathbf{R}^{3}\right)$ and $\delta\left(x-x_{i}\right)$ are the $\delta$ measures at the points $x_{i} \cdot \lambda \sum_{i=1}^{n} \delta\left(x-x_{i}\right)$ is a measure with support on the finite set $\left\{x_{1}, \ldots, x_{n}\right\}, x_{i} \in \mathbf{R}^{3}$. (3.1) is of course entirely formal and does not make sense as an operator in $L_{2}\left(\mathbf{R}^{3}\right)$. Formally the corresponding bilinear form is given by

$$
\int_{\mathbf{R}^{3}}|\nabla f|^{2}(x) d x-\lambda \sum_{i=1}^{n}\left|f\left(x_{i}\right)\right|^{2}
$$

which at least is a densely defined bilinear form on $L_{2}\left(\mathbf{R}^{3}\right)$, but it is not closable and therefore it does not define any operator. Introducing the Fourier rapsorm, (3.2) takes the form

$$
\int_{\mathbf{R}^{3}} p^{2}|\tilde{f}(p)|^{2} d p-\lambda \sum_{j=1}^{n}\left|\int_{\mathbf{R}^{3}} \tilde{f}(p) e^{i x_{j} p} d p\right|^{2} .
$$

We are naturally led to first study the following bilinear form on the standard Hilbert space $L_{2}\left(\mathbf{R}^{3}\right)$ :

$$
\left(\tilde{f}, p^{2} \tilde{f}\right)-\lambda \sum_{j=1}^{n}\left|\int_{|p|<\omega} \tilde{f}(p) e^{i x_{j} p} d p\right|^{2}
$$

where $\lambda_{j}$ and $\omega$ are in $\mathbf{R}$ and $p^{2}$ is the selfadjoint operator on $L_{2}\left(\mathbf{R}^{3}\right)$ given by multiplication by $p^{2}=p_{1}^{2}+p_{2}^{2}+p_{3}^{2}$. (3.4) is obviously a closed semibounded form on $L_{2}\left(R^{3}\right)$ and the corresponding selfadjoint operator is given by 


$$
H=p^{2}-\lambda \sum_{j=1}^{n} \psi_{x_{j}} \otimes \psi_{x_{j}}
$$

and $\psi_{x}(p)=\chi_{\omega}(p) e^{i x p}$ where $\chi_{\omega}$ is the characteristic function of the ball $|p| \leqslant \omega$ and we write $\varphi \otimes \psi$ for the operator

$$
(\varphi \otimes \psi) f=(\psi, f) \varphi
$$

whenever $\varphi$ and $\psi$ are in the Hilbert space $L_{2}\left(\mathbf{R}^{3}\right)$. (3.5) is then a perturbation of $p^{2}$ by an operator of finite rank $n$.

Set now

$$
V=\sum_{j=1}^{n} \psi_{x_{j}} \otimes \psi_{x_{j}}
$$

Since $V$ is bounded we have for $m$ big enough

$$
\begin{aligned}
& \left(H+m^{2}\right)^{-1} \\
& =\left(p^{2}+m^{2}\right)^{-1 / 2}\left(1-\lambda\left(p^{2}+m^{2}\right)^{-1 / 2} V\left(p^{2}+m^{2}\right)^{-1 / 2}\right)^{-1}\left(p^{2}+m^{2}\right)^{-1 / 2}
\end{aligned}
$$

Set now

$$
A=\left(p^{2}+m^{2}\right)^{-1 / 2} V\left(p^{2}+m^{2}\right)^{-1 / 2}
$$

then

$$
A^{k}=\left(p^{2}+m^{2}\right)^{-1 / 2} V\left(p^{2}+m^{2}\right)^{-1} \ldots V\left(p^{2}+m^{2}\right)^{-1} V\left(p^{2}+m^{2}\right)^{-1 / 2} .
$$

Now define the $n \times m$ matrix $g_{i j}$ by

$$
g_{i j}=\left(\psi_{x_{i}},\left(p^{2}+m^{2}\right)^{-1} \psi_{x_{j}}\right)
$$

then

$$
g_{i j}=\int_{|p|<\omega} e^{i\left(x_{j}-x_{i}\right) p} \frac{d p}{p^{2}+m^{2}} \equiv G_{\omega}\left(x_{i}-x_{j}\right)
$$

and

$$
A^{k}=\left(p^{2}+m^{2}\right)^{-1 / 2}\left[\sum_{j_{1}, \ldots, j_{k}} \psi_{x_{j_{1}}} g_{j_{1} j_{2}} g_{j_{2} j_{3}} \ldots g_{j_{k-1} j_{k}} \otimes \psi_{x_{j_{k}}}\right]\left(p^{2}+m^{2}\right)^{-1 / 2}
$$

or

$$
A^{k}=\left(p^{2}+m^{2}\right)^{-1 / 2}\left[\sum_{i, j} \psi_{x_{i}}\left(g^{k-1}\right)_{i j} \otimes \psi_{x_{j}}\right]\left(p^{2}+m^{2}\right)^{-1 / 2}
$$

where $\left(g^{k-1}\right)_{i j}$ is the $k-1$ power of the matrix $g_{i j}$. Since $A$ is a bounded 
operator we have for $|\lambda| \leqslant\|A\|^{-1}$ that

$$
(1-\lambda A)^{-1}=\sum_{k=0}^{\infty} \lambda^{k} A^{k}
$$

which by (3.14) is equal to

$$
1+\lambda\left(p^{2}+m^{2}\right)^{-1 / 2}\left[\sum_{i, j} \psi_{x_{i}}\left(\sum_{k=0}^{\infty} \lambda^{k} g^{k}\right)_{i j} \otimes \psi_{x_{j}}\right]\left(p^{2}+m^{2}\right)^{-1 / 2}
$$

Now

$$
\lambda \sum_{k=0}^{\infty} \lambda^{k} g^{k}=\lambda(1-\lambda g)^{-1}=\left(\lambda^{-1}-g\right)^{-1} .
$$

Hence we have that

$$
(1-\lambda A)^{-1}=1+\left(p^{2}+m^{2}\right)^{-1 / 2}\left[\sum_{i, j} \psi_{x_{i}}\left(\lambda^{-1}-g\right)_{i j}^{-1} \otimes \psi_{x_{j}}\right]\left(p^{2}+m^{2}\right)^{-1 / 2} \text {. }
$$

Thus from (3.8) we get

$$
\begin{aligned}
\left(p^{2}\right. & \left.+m^{2}-\lambda V\right)^{-1} \\
& =\left(p^{2}+m^{2}\right)^{-1}+\left(p^{2}+m^{2}\right)^{-1}\left[\sum_{i, j}^{n}\left(\lambda^{-1}-g\right)_{i j}^{-1} \psi_{x_{i}} \otimes \psi_{x_{j}}\right]\left(p^{2}+m^{2}\right)^{-1} .
\end{aligned}
$$

Since (3.19) holds for all $\lambda$ in the complex disk $|\lambda| \leqslant\|A\|^{-1}$ it holds also by analytic continuation for all $\lambda$ such that $\lambda^{-1}$ is not an eigenvalue of $g$. Hence (3.19) holds for all $\lambda \in \mathbf{R}$ with the exception of at most $n$ real points, namely the points where $\lambda^{-1}$ is an eigenvalue of the symmetric $n \times m$ matrix $g$.

Up to now in this paragraph all our considerations have been standard. Let us now consider the nonstandard Hilbert space ${ }^{*} L_{2}\left(\mathbf{R}^{3}\right)$ and the form

$$
\left(f, p^{2} f\right)-\lambda \sum_{j=1}^{n}\left|\int_{|p|<\omega} f(p) e^{i x_{j} p} d p\right|^{2}
$$

where $\lambda$ and $\omega$ are in ${ }^{*} \mathbf{R}$ and $\omega$ is taken to be infinite positive, i.e. positive and not near standard. By the transfer principle (3.20) defines a closed form on ${ }^{*} L_{2}\left(\mathbf{R}^{3}\right)$ and, again by the transfer principle, the corresponding selfadjoint operator $H$ satisfies again (3.19) except at most for $n$ real values of $\lambda$. Hence we have that

$$
\left(H+m^{2}\right)^{-1}=\left(p^{2}+m^{2}\right)^{-1}+\sum_{i j}^{n}\left(\lambda^{-1}-g\right)_{i j}^{-1} \varphi_{x_{i}} \otimes \varphi_{x_{j}}
$$


where

$$
g_{i j}=\int_{|p|<\omega} e^{i\left(x_{j}-x_{i}\right) p} \frac{d p}{p^{2}+m^{2}}=G_{\omega}\left(x_{i}-x_{j}\right)
$$

and

$$
\varphi_{x}=\chi_{\omega}(p) e^{i x p} /\left(p^{2}+m^{2}\right)
$$

where $\chi_{\omega}$ is the characteristic function for the nonstandard ball $|x|<\omega$. Let us now assume that $x_{i} \in \mathbf{R}$ and $x_{i} \neq x_{j}$ for $i \neq j$. We want to choose $\lambda$ such that $H$ is near standard, i.e. that $\left(H+m^{2}\right)^{-1}$ is near standard. From (3.23) we have that $\varphi_{x}$ is near standard and in fact

$$
\text { st } \varphi_{x}=e^{i x p} /\left(p^{2}+m^{2}\right)
$$

since $\omega$ is infinite. From (3.22) we see that $g_{i j}$ for $i \neq j$ is near standard and in fact if $i \neq j$ then

$$
\text { st } g_{i j}=\int_{\mathbf{R}^{3}} e^{i\left(x_{j}-x_{i}\right) p} \frac{d p}{p^{2}+m^{2}}=G\left(x_{i}-x_{j}\right)
$$

with

$$
G(x)=\frac{1}{4 \pi|x|} e^{-m|x|}
$$

On the other hand

$$
g_{i i}=G_{\omega}(0)=\int_{|p|<\omega} \frac{d p}{p^{2}+m^{2}}
$$

is not near standard. However let us choose $\lambda \in{ }^{*} \mathbf{R}$ such that

$$
\lambda^{-1}=G_{\omega}(0)+\alpha
$$

with $\alpha \in \mathbf{R}$, then $\lambda^{-1} 1-g$ is near standard, and if we set

$$
\tilde{g}_{i j}= \begin{cases}0 & \text { for } i=j \\ G\left(x_{i}-x_{j}\right) & \text { for } i \neq j\end{cases}
$$

then

$$
\operatorname{st}\left(\lambda^{-1}-g\right)=\alpha-\tilde{g} .
$$

Since the exceptional set for (3.21) consists of the values of $\lambda$ for which $\lambda^{-1}$ is an eigenvalue of $g$ we get that this exceptional set corresponds to the set of $\alpha$ for which $\alpha$ is an eigenvalue of $\tilde{g}$. Hence with this choice of $\lambda$ we have that $\left(H+m^{2}\right)^{-1}$ is near standard and therefore by definition that $H$ is near standard. We have now proved the following theorem, expressed in the Fourier transformed representation. 
THEOREM 3.1. Let $\delta_{\omega}(x)$ be the Fourier transform of the characteristic function $\chi_{\omega}$ of the nonstandard ball $|x|<\omega$ with $\omega$ infinite. Consider the closed form

$$
-(f, \Delta f)-\lambda \sum_{j=1}^{n}\left|\left(f, \delta_{\omega}\left(\cdot-x_{j}\right)\right)\right|^{2}
$$

on the nonstandard Hilbert space ${ }^{*} L_{2}\left(\mathbf{R}^{3}\right)$, where $\left\{x_{1}, \ldots, x_{n}\right\}$ is a finite set in $\mathbf{R}^{3}$ so that $x_{i} \neq x_{j}$ if $i \neq j, \lambda \in{ }^{*} \mathbf{R}$ and $\Delta$ is the selfadjoint Laplacian in ${ }^{*} L_{2}\left(\mathbf{R}^{3}\right)$. Let $G(x)=e^{-m|x|} / 4 \pi|x|$ for $x \in \mathbf{R}$ and let $g=g_{i j}$ be the standard $n \times m$ matrix

$$
g_{i j}= \begin{cases}0 & \text { if } i=j, \\ G\left(x_{i}-x_{j}\right) & \text { if } i \neq j,\end{cases}
$$

and let $\lambda_{\omega}(\alpha)$ be given by

$$
\lambda_{\omega}^{-1}(\alpha)=\int_{|p|<\omega} \frac{d p}{p^{2}+\omega^{2}}+\alpha .
$$

If $H$ is the selfadjoint operator corresponding to the closed form above then $H$ is near standard if $\lambda=\lambda_{\omega}(\alpha)$ with $\alpha \in \mathbf{R}$. Moreover we have that if $m \in \mathbf{R}$ and $m>0$ then, for any $\alpha$ not an eigenvalue of $g$,

$$
\operatorname{st}\left(H+m^{2}\right)^{-1}=\left(-\Delta+m^{2}\right)^{-1}+\sum_{i j}(\alpha-g)_{i j}^{-1} G_{x_{i}} \otimes G_{x_{j}}
$$

where $G_{x_{i}}(x)=G\left(x-x_{i}\right)$. If we set $H_{\alpha}=$ st $H$ for $\lambda=\lambda_{\omega}(\alpha)$ we see that $-m^{2}$ is an eigenvalue for $H_{\alpha}$ if and only if $\alpha$ is an eigenvalue for $g$. Thus $H_{\alpha}$ has at most $n$ eigenvalues.

REMARK. In the case $n=1$, the family $H_{\alpha}$ coincides with the family of Theorem 2.3.

4. Singular Sturm-Liouville theory. Let $\boldsymbol{A}$ be the selfadjoint Sturm-Liouville operator with Dirichlet boundary conditions given by

$$
A u=-\left(p u^{\prime}\right)^{\prime}+q u
$$

on the standard Hilbert space $L_{2}([a, b], d x)$, where $p$ and $q$ are smooth real functions such that $p \geqslant \varepsilon>0$ and $q \geqslant 0$. The regular Sturm-Liouville theory then tells us that $A$ has discrete spectrum $0<\lambda_{0}<\lambda_{1}<\cdots$, consisting of simple eigenvalues such that

$$
\sum_{i=0}^{\infty} \lambda_{i}^{-1}<\infty
$$

Moreover if $A u_{n}=\lambda_{n} u_{n}$ then $u_{n}$ has $n+2$ zeros in the interval $[a, b]$ and the zeros of $u_{n+1}$ separate the zeros of $u_{n}$. We are now interested in extending these results to the case where $p$ and $q$ are "singular functions", in particular 
the case where $q$ is a bounded measure. In this case in particular (4.1) does not make sense as a differential operator in $L_{2}([a, b])$ and we shall have to give an alternative definition of $A$. To do this it will be useful to consider the corresponding nonstandard Sturm-Liouville operator in ${ }^{*} L_{2}([a, b])$, with Dirichlet boundary conditions,

$$
\tilde{A} u=-\left(p u^{\prime}\right)^{\prime}+q u
$$

with $p, q$ nonstandard but smooth functions, resp. measures. By analyzing the nonstandard problem, we will be able to handle the standard problem. In fact the nonstandard problem, being given in terms of a (nonstandard) differential operator has the corresponding properties as the standard regular SturmLiouville problem, and by using this we are able to deduce corresponding conclusions on the singular (standard) Sturm-Liouville problem we are interested in.

We first examine closer the standard operator

$$
A_{0} u=-\left(p u^{\prime}\right)^{\prime}
$$

with Dirichlet boundary conditions on $L_{2}([a, b])$, and where $p$ is now a real measurable nonnegative (not necessarily strictly positive or smooth) function. Let

$$
v_{1}(x)=\int_{a}^{x} \frac{d z}{p(z)} \text { and } v_{2}(x)=\int_{x}^{b} \frac{d z}{p(z)} .
$$

We assume that $1 / p$ is in $L_{1}([a, b])$ so that $v_{i}, i=1,2$, are bounded continuous functions. Let

$$
G_{0}(x, y)=\frac{1}{K} \begin{cases}v_{1}(x) v_{2}(y), & x \leqslant y, \\ v_{1}(y) v_{2}(x), & y \leqslant x,\end{cases}
$$

where

$$
K=p(x)\left(v_{1}^{\prime}(x) v_{2}(x)-v_{1}(x) v_{2}^{\prime}(x)\right) .
$$

$K$ is independent of $x$ and $G_{0}(x, y)$ is the kernel of $G_{0}=A_{0}^{-1}$. We see that under the assumption that $p^{-1} \in L_{1}([a, b])$ then $G_{0}(x, y)$ is a bounded continuous function. From (4.6) we have

$$
K=\int_{a}^{b} \frac{d z}{p(z)}
$$

and since $p \geqslant 0$ by assumption we get $K \geqslant 0$, hence

$$
G_{0}(x, y) \geqslant 0 \text {. }
$$

$A_{0}$ has discrete spectrum consisting of simple eigenvalues $\lambda_{i}^{0}, i=0,1 \ldots$ Since the operator $G_{0}$ is of trace class, $G_{0}(x, y)$ being bounded and continuous, with 


$$
\operatorname{tr} G_{0}=\int_{a}^{b} G(x, x) d x
$$

we have

$$
\sum_{i=0}^{\infty}\left(\lambda_{i}^{0}\right)^{-1}=\left(\int_{a}^{b} \frac{d z}{p(z)}\right)^{-1} \int_{a}^{b}\left[\int_{a}^{x} \frac{d z}{p(z)} \int_{x}^{b} \frac{d z}{p(z)}\right] d x<\infty .
$$

From this we get in particular, if we order the eigenvalues in such a way that $\lambda_{0}^{0}<\lambda_{1}^{0}<\lambda_{2}^{0}<\cdots$,

$$
\left(\lambda_{0}^{0}\right)^{-1} \leqslant(b-a) \int_{a}^{b} \frac{d z}{p(z)} .
$$

Hence

$$
0<\lambda_{0}^{0}<\lambda_{1}^{0}<\cdots
$$

We shall now consider perturbations of $A_{0}$. Let first $q \in L_{\infty}([a, b])$ and $q \geqslant 0$, then the selfadjoint operator

$$
A u=-\left(p u^{\prime}\right)^{\prime}+q u
$$

with Dirichlet boundary conditions satisfies

$$
A \geqslant A_{0}
$$

so that if $\lambda_{i}$ are the eigenvalues of $A$ then $\lambda_{i}>\lambda_{i}^{0}$ and in particular

$$
\lambda_{0}^{-1} \leqslant(b-a) \int_{a}^{b} \frac{d z}{p(z)} .
$$

Moreover if $u_{i}$ is the eigenfunction corresponding to $\lambda_{i}$, i.e.

$$
A u_{i}=\lambda_{i} u_{i}
$$

then, by the methods of the ordinary (singular) Sturm-Liouville theory, we have that $u_{n}$ has exactly $n+2$ zeros $\alpha_{n}^{(k)}, k=1, \ldots, n+2$, in $[a, b]$ and the zeros of $u_{n+1}$ separate those of $u_{n}$, i.e.

$$
\alpha_{n}^{(k)}<\alpha_{n+1}^{(k+1)}<\alpha_{n}^{(k+1)}
$$

for $k \neq 0, n+2$. Now we have

$$
-\left(p u_{n}^{\prime}\right)^{\prime}+q u_{n}=\lambda_{n} u_{n}
$$

and

$$
u_{n}\left(\alpha_{n}^{(k)}\right)=u_{n}\left(\alpha_{n}^{(k+1)}\right)=0
$$

so that $\lambda_{n}$ is an eigenvalue of the Sturm-Liouville operator (4.17) on $L_{2}\left(\left[\alpha_{n}^{(k)}, \alpha_{n}^{(k+1)}\right]\right)$, with Dirichlet boundary conditions in $\alpha_{n}^{(k)}$ and $\alpha_{n}^{(k+1)}$, with correspondent eigenfunction $u_{n}$. Since $u_{n}$ has no zeros in $\left(\alpha_{n}^{(k)}, \alpha_{n}^{(k+1)}\right)$ we see that $\lambda_{n}$ is the lowest eigenvalue of this Sturm-Liouville operator. Thus from (4.15) we have 


$$
\lambda_{n}^{-1} \leqslant\left(\alpha_{n}^{(k+1)}-\alpha_{n}^{(k)}\right) \int_{\alpha_{n}^{(k)}}^{\alpha_{n}^{(k+1)}} \frac{d z}{p(z)} .
$$

This gives a lower bound on the distance $\alpha_{n}^{(k+1)}-\alpha_{n}^{(k)}$ between consecutive zeros of $u_{n}$.

Let us now on the other hand expand $\left(A_{0}-\lambda q\right)^{-1}$ in powers of $\lambda$, which is allowed by $q \in L_{\infty}$, for $|\lambda|<\left\|G_{0}\right\|_{\infty}^{-1}\|q\|^{-1}$. We get

$$
\left(A_{0}-\lambda q\right)^{-1}=G_{0}\left(1+\lambda q G_{0}\right)^{-1}=\sum_{n=0}^{\infty} \lambda^{n} G_{0} q G_{0} \ldots q G_{0} .
$$

The kernel of $G_{0} q G_{0} \ldots q G_{0}$ is given by

$$
\begin{aligned}
\left(G_{0} q G_{0} \ldots q G_{0}\right)(x, y) & \\
& =\int \ldots \int G_{0}\left(x, x_{1}\right) G_{0}\left(x_{1}, x_{2}\right) \ldots G_{0}\left(x_{n}, y\right) \prod_{i=1}^{n} q\left(x_{i}\right) d x_{i} .
\end{aligned}
$$

We now remark that the right-hand side of (4.21) also makes sense if $q$ is replaced by a positive bounded measure $\mu$. Hence we can define the kernel

$$
\begin{aligned}
\left(G_{0} \mu G_{0} \ldots \mu G_{0}\right)(x, y) & \\
& \equiv \int \cdots \int G_{0}\left(x, x_{1}\right) \ldots G_{0}\left(x_{n}, y\right) d \mu\left(x_{1}\right) \ldots d \mu\left(x_{n}\right)
\end{aligned}
$$

We observe that from (4.22) we have

$$
\left\|G_{0} \mu G_{0} \ldots \mu G_{0}\right\|_{\infty} \leqslant\left\|G_{0}\right\|^{n+1}\|\mu\|^{n} .
$$

This gives us the possibility to define the operator $\left(A_{0}-\lambda \mu\right)^{-1}$ as the operator in $L_{2}([a, b])$ with kernel

$$
\left(A_{0}-\lambda \mu\right)^{-1}(x, y)=\sum_{n=0}^{\infty} \lambda^{n}\left(G_{0} \mu G_{0} \ldots \mu G_{0}\right)(x, y),
$$

for $|\lambda|<\left\|G_{0}\right\|_{\infty}^{-1}\|\mu\|^{-1}$. In fact the series (4.24) converges, by (4.23), uniformly. Since moreover the kernel in (4.22) is continuous we get that $\left(A_{0}-\lambda \mu\right)^{-1}$ is continuous, hence (4.24) defines, for $\lambda$ real and such that $|\lambda|<\left\|G_{0}\right\|_{\infty}^{-1}\|\mu\|^{-1}$, a bounded symmetric operator in $L_{2}([a, b])$ with continuous kernel. For $\lambda \geqslant 0$ we get from (4.24) that $\left(A_{0}-\lambda \mu\right)^{-1}$ is positive, and in fact

$$
\left(A_{0}-\lambda \mu\right)^{-1} \geqslant G_{0}
$$

Since $G_{0}=A_{0}^{-1}$ is invertible, we get from (4.24) that, for $|\lambda|<\left\|G_{0}\right\|_{\infty}^{-1}\|\mu\|^{-1}$, $\left(A_{0}-\lambda \mu\right)^{-1}$ is invertible, with positive inverse

$$
A_{0}-\lambda \mu \geqslant 0 \text {. }
$$

From this we get that the form $(f, \mu f)=\int|f|^{2} d \mu$ is bounded in the following way 


$$
\lambda_{0}(f, \mu f) \leqslant\left(f, A_{0} f\right)
$$

for any $f \in D\left(A_{0}^{1 / 2}\right)$ and $0 \leqslant \lambda_{0}<\left\|G_{0}\right\|_{\infty}^{-1}\|\mu\|^{-1}$. This implies, since $\mu$ is a positive measure, that

$$
\lambda_{0}(f, \mu f) \leqslant\left(f,\left(A_{0}+\lambda \mu\right) f\right)
$$

for any positive $\lambda$. From this we get that $\left(f,\left(A_{0}+\lambda \mu\right) f\right)$ is a closed positive form for any positive $\lambda$ and thus defines a unique selfadjoint operator $A_{0}+\lambda \mu$, for any positive $\lambda$.

Hence for any bounded positive measure $\mu$ we have defined a selfadjoint operator $A=A_{0}+\mu$. From the obvious fact that

$$
A \geqslant A_{0}
$$

and the fact that $A_{0}$ has discrete spectrum we have that $A$ has discrete spectrum. If $\lambda_{n}$ are the eigenvalues with corresponding eigenfunctions $u_{n}$, so that

$$
A u_{n}=\lambda_{n} u_{n}
$$

then by (4.29) and (4.10), we have $\Sigma_{n} \lambda_{n}^{-1}<\infty$. Now for any element $f$ in $D\left(A_{0}^{1 / 2}\right)$ we have

$$
|f(y)-f(x)|=\left|\int_{x}^{y} f^{\prime}(z) d z\right|=\left|\int_{x}^{y} f^{\prime}(z) \sqrt{p(z)} \frac{1}{\sqrt{p(z)}} d z\right|,
$$

hence by Schwarz inequality

$$
|f(y)-f(x)| \leqslant\left(\int_{x}^{y} f^{\prime}(z)^{2} p(z) d z\right)^{1 / 2}\left(\int_{x}^{y} \frac{1}{p(z)} d z\right)^{1 / 2} .
$$

From this it follows that $f \in D\left(A_{0}^{1 / 2}\right)$ is continuous and since by (4.28) we have $D\left(A^{1 / 2}\right) \subset D\left(A_{0}^{1 / 2}\right)$, it follows from this that all eigenfunctions of $A$ are continuous, i.e. $u_{n}$ are continuous functions for all $n$.

We can ask ourselves now whether the results of the ordinary Sturm-Liouville theory about the simplicity of the eigenvalues $\lambda_{n}$, the number of zeros of $u$ and the separation theorem that the zeros of $u_{n+1}$ should separate those of $u_{n}$ carry over to our singular situations. This is especially interesting since in the ordinary proof of this type of results the fact that the Sturm-Liouville operator is a second order differential operator is used in an essential way, whereas our operator $A_{0}+\mu$ is not a differential operator in the ordinary sense when $\mu$ is not a function.

We first observe that for the corresponding nonstandard problem we have indeed a result of the type we want. Consider namely the nonstandard Hilbert space ${ }^{*} L_{2}([a, b])$ and the corresponding nonstandard Sturm-Liouville operator with Dirichlet boundary conditions

$$
\tilde{A} \tilde{u}=-\left(p \tilde{u}^{\prime}\right)^{\prime}+q \tilde{u}
$$


in ${ }^{*} L_{2}([a, b])$. We assume that $p$ is a nonstandard function satisfying the conditions $1 / p \in{ }^{*} L_{1}([a, b])$ and $p>0$, which are the nonstandard correspondent of those we required for $p$ in the standard problem. About $q$ we assume that it is a smooth nonstandard positive function. Then $\tilde{A}$ is a well defined selfadjoint operator in ${ }^{*} L_{2}([a, b])$ and by the transfer principle we have that $\tilde{A}$ has discrete spectrum, with simple eigenvalues $0<\tilde{\lambda}_{0}<\tilde{\lambda}_{1}$ $<\cdots<\tilde{\lambda}_{n}<\cdots, n \in{ }^{*} N$ and if $\tilde{u}_{n}$ is the eigenfunction to the eigenvalue $\tilde{\lambda}_{n}$, then $\tilde{u}_{n}$ has exactly $n+2$ zeros and the zeros of $\tilde{u}_{n+1}$ separate those of $\tilde{u}_{n}$.

Consider now the special case where $q$ is a smooth nonstandard realization of the standard measure $\mu$, e.g.

$$
q(x)=\int \delta_{\varepsilon}(x-y) d \mu(y),
$$

where $\delta_{\varepsilon}(x)=\left({ }^{*} \varphi / \varepsilon\right)(x / \varepsilon)$, with $\varepsilon$ positive and infinitesimal and $\varphi(x)$ a positive $C_{0}^{\infty}$-function of compact support such that $\int \varphi(x) d x=1$. Computing $\tilde{A}^{-1}$ in the corresponding way as we computed $\left(A_{0}+q\right)^{-1}$ in the regular case, we get that $\tilde{A}^{-1}$ is near standard and in fact

$$
\text { st } \tilde{A}^{-1}=\left(A_{0}+\mu\right)^{-1} \text {. }
$$

Let now $u_{n}$ be the eigenfunction in $L_{2}([a, b])$ for $A_{0}+\mu$ corresponding to the eigenfunction $\tilde{u}_{n}$ for $\tilde{A}$ in ${ }^{*} L_{2}([a, b])$, then by (4.35) we have

$$
u_{n}=\text { st } \tilde{u}_{n} \text {. }
$$

Since $\tilde{u}_{n}$ has exactly $n+2$ zeros $\tilde{\alpha}_{n}^{(k)}, k=0, \ldots, n+1$ and $u_{n}$ is continuous, we see that $u_{n}$ has at least $n+2$ zeros, namely the points $\alpha_{n}^{(k)}=$ st $\tilde{\alpha}_{n}^{(k)}$.

We shall now show that these zeros have a nonvanishing standard distance from each other. If we call $\lambda_{n}$ the eigenvalues of $A_{0}+\mu$ corresponding to the eigenfunction $u_{n}$ we have, for $n \in N$, by (4.35), that

$$
\lambda_{n}=\text { st } \tilde{\lambda}_{n} \text {. }
$$

From the transfer principle we have then from (4.19) that

$$
\left(\tilde{\alpha}_{n}^{(k+1)}-\tilde{\alpha}_{n}^{(k)}\right) \int_{\tilde{\alpha}_{n}^{(k)}}^{\tilde{\alpha}_{n}^{(k+1)}} \frac{d z}{p(z)} \geqslant \tilde{\lambda}_{n}^{-1}
$$

from which it follows by taking the standard part that

$$
\left(\alpha_{n}^{(k+1)}-\alpha_{n}^{(k)}\right) \int_{\alpha_{n}^{(k)}}^{\alpha_{n}^{(k+1)}} \frac{d z}{p(z)} \geqslant \lambda_{n}^{-1}>0,
$$

so that the $\alpha_{n}^{(k)}$ are distinct zeros for $u_{n}$. We are now going to show that $u_{n}$ has no more than these $n+2$ zeros. Analogously as in the usual standard regular case we consider the initial value problem associated with our nonstandard Sturm-Liouville Dirichlet problem, namely the differential equation

$$
-\left(p \tilde{u}^{\prime}\right)^{\prime}+q \tilde{u}=\tilde{\lambda} \tilde{u}
$$


over ${ }^{*} \mathbf{R}$ with $q$ given as before by (4.34). By the transfer principle we have that the initial value problem (4.40) with

$$
\tilde{u}(a)=\tilde{\alpha}, \quad \tilde{u}^{\prime}(a)=\tilde{\beta}
$$

has the solution

$$
\tilde{u}(x)=\int_{a}^{x} \frac{d y}{p(y)} \int_{a}^{y}(q(z)-\tilde{\lambda}) \tilde{u}(z) d z+p(a) \tilde{\beta} \int_{a}^{x} \frac{d y}{p(y)}+\tilde{\alpha} .
$$

Hence if $\tilde{\alpha}, \tilde{\beta}$ and $\tilde{\lambda}$ are near standard then $u=$ st $\tilde{u}$ satisfies

$$
\begin{aligned}
u(x)= & \int_{a}^{x} \frac{d y}{p(y)}\left[\int_{a}^{y} u(z) d \mu(z)-\lambda \int_{a}^{y} u(z) d z\right] \\
& +p(a) \beta \int_{a}^{x} \frac{d y}{p(y)}+\alpha,
\end{aligned}
$$

where $\alpha, \beta$ and $\lambda$ are the standard parts of $\tilde{\alpha}, \tilde{\beta}$ and $\tilde{\lambda}$ respectively. We are going now to show that (4.43), looked upon as an integral equation for $u$, has a unique solution. In fact set

$$
T u=\int_{a}^{x} \frac{d y}{p(y)}\left[\int_{a}^{y} u(z) d \mu(z)-\lambda \int_{a}^{y} u(z) d z\right]
$$

Then

$$
|T u(x)| \leqslant \int_{a}^{c} \frac{d y}{p(y)}[\mu([a, c])+|\lambda|(c-a)]\|u\|_{\infty},
$$

so that $T$ as an operator on $C([a, c])$ satisfies

$$
\|T\| \leqslant \int_{a}^{c} \frac{d y}{p(y)}[\mu([a, c])+|\lambda|(c-a)] .
$$

Hence if we choose $c$ so small that

$$
\int_{a}^{c} \frac{d y}{p(y)}[\mu([a, c])+|\lambda|(c-a)]<1
$$

then, by the contracting map principle, (4.43) on the interval $[a, c]$ has a unique solution $u$. Repeating now the same considerations for an interval $[c, d]$, with initial conditions at $c$ given by the corresponding values $u(c), u^{\prime}(c)$ of $\alpha, \beta$ we may continue this further and since $1 / p \in L_{1}([a, b])$ we actually get that (4.43) has a unique solution in the interval $[a, b]$ and the solution is in $C[a, b]$. It follows easily that $\lambda$ is an eigenvalue of $A_{0}+\mu$ if and only if (4.43) with $\alpha=0, \beta \neq 0$ has a solution $u \neq 0$, so that for this solution we have that $u(b)=0$. But by the uniqueness of the solution of (4.43) we then have that $u$ is the unique solution with $u(a)=0, u(b)=0$, hence $u$ is also the unique eigenfunction for $A_{0}+\mu$ to the eigenvalue $\lambda$. Thus the family of solutions of

$$
\left(A_{0}+\mu\right) u_{n}=\lambda_{n} u_{n}
$$


for a fixed eigenvalue $\lambda_{n}$ of $A_{0}+\mu$ is one-dimensional. Therefore we have proven that the eigenvalues $\lambda_{n}$ are simple and we have in fact

$$
0<\lambda_{0}<\lambda_{1}<\cdots
$$

i.e. all $\lambda_{n}$ are different.

Take now $\alpha=0$ and $\beta=1$ in (4.43), then

$$
u(x)=\int_{a}^{x} \frac{d y}{p(y)}\left[\int_{a}^{y} u(z) d \mu(z)-\lambda \int_{a}^{y} u(z) d z\right]+p(a) \int_{a}^{x} \frac{d y}{p(y)} .
$$

We see that for $\lambda=0$ we have $u(x) \geqslant 0$ for all $x$, because $u(0)=0$ and $u^{\prime}(0)=1$, so that for $\lambda=0$ we have

$$
u(x) \geqslant p(a) \int_{a}^{x} \frac{d y}{p(y)}>0 .
$$

From (4.50) we also get that if $u$ is positive in the interval $[a, x]$ then $u(x)$ is a monotone decreasing function of $\lambda$. Call $c(\lambda)$ the first zero of $u(x)$ situated at the right of $a$, then $c(\lambda)$ is a monotone decreasing function of $\lambda$. Moreover $\lambda_{0}$, the first eigenvalue of $A_{0}+\mu$, is the unique solution of $c(\lambda)=b$. This however implies that the eigenfunction $u_{0}$ of $A_{0}+\mu$ corresponding to the eigenvalue $\lambda_{0}$ is strictly positive in the interior of the interval $[a, b]$. Applying now these considerations to the restriction of $u_{n}$ to the interval $\left[\alpha_{n}^{(k)}, \alpha_{n}^{(k+1)}\right]$ we get again that $u_{n}$ is different from zero in the open interval $\left(\alpha_{n}^{(k)}, \alpha_{n}^{(k+1)}\right)$. This then shows that $u_{n}$ has exactly $n+2$ zeros. Moreover from the fact established above from the transfer principle that $\tilde{u}_{n}$ changes sign in consecutive intervals (being continuous and having zeros between $\tilde{\alpha}_{n+1}^{(k)}$ and $\tilde{\alpha}_{n+1}^{(k+1)}$ ), we have that its standard part $u_{n}$ also changes sign in consecutive intervals. Since $\alpha_{n}^{(k)}$ is the standard part of $\tilde{\alpha}_{n}^{(k)}$, we have that the $\alpha_{n+1}^{(k)}$ separate the $\alpha_{n}^{(k)}$. Hence we have proven all results we were interested in, corresponding to those of the standard regular Sturm-Liouville problem. We formulate this in the following

THEOREM 4.1. Let $p$ be a positive function on $[a, b]$ such that $p^{-1} \in$ $L_{1}([a, b])$ and let $\mu$ be a positive bounded measure on the interval $[a, b]$. Then the form

$$
\int_{a}^{b} p(x) u^{\prime}(x)^{2} d x+\int_{a}^{b} u(x)^{2} d \mu(x)
$$

with zero boundary conditions is a closed positive form on $L_{2}[a, b]$ and thus defines a positive selfadjoint operator $A=A_{0}+\mu$. $A$ has discrete spectrum consisting of simple eigenvalues $0<\lambda_{0}<\lambda_{1}<\cdots$, such that $\Sigma \lambda_{i}^{-1}<\infty$, and the eigenfunction $u_{n}$ corresponding to the eigenvalue $\lambda_{n}$ is continuous on $[a, b]$ and has exactly $n+2$ zeros on $[a, b]$ and changes sign as one passes the zeros. Moreover the zeros of $u_{n+1}$ separate the zeros of $u_{n}$. 
Let $A$ be the nonstandard Sturm-Liouville operator given by the nonstandard differential operator

$$
\tilde{A} u=-\left({ }^{*} p \tilde{u}^{\prime}\right)^{\prime}+q \tilde{u}
$$

on ${ }^{*} L_{2}([a, b])$ with Dirichlet boundary conditions, such that $q$ is the smooth positive nonstandard function given by $q=\delta_{\varepsilon} *\left({ }^{*} \mu\right)$, where $\delta_{\varepsilon}(x)=$ $\left({ }^{*} \varphi / \varepsilon\right)(x / \varepsilon)$, with $\varepsilon$ positive and infinitesimal and ${ }^{*} \varphi$ the nonstandard extension of a smooth positive standard function $\varphi$ such that $\int \varphi(x) d x=1$. Then $\tilde{A}$ is a positive selfadjoint near standard operator on ${ }^{*} L_{2}([a, b])$ and $A=$ st $\tilde{A}$ in the sense that $A^{-1}=$ st $\tilde{A}^{-1}$. Moreover if $\tilde{\lambda}_{n}$ and $\tilde{u}_{n}$ for $n \in{ }^{*} N$ are the eigenvalues and eigenfunctions of $\tilde{A}$ then for $n \in N$, we have $\lambda_{n}=$ st $\tilde{\lambda}_{n}$ and $u_{n}=$ st $\tilde{u}_{n}$.

ACKNOWLEDGements. We are grateful to Professor Edward Nelson for sending us a Preprint of his invited address at the A.M.S. 1976 Summer Meeting, the discussion of which has stimulated our work. We would also like to thank the participants of the seminar on nonstandard analysis held at the Mathematics Institute of Oslo University during the academic year 1976-1977 for creating an exciting atmosphere and contributing to the discussions. We are also grateful to the Referee for very valuable comments.

\section{REFERENCES}

1. A. Robinson, Non standard analysis, North-Holland, Amsterdam, 1970.

2. K. D. Stroyan and W. A. J. Luxemburg, Introduction to the theory of infinitesimals, Academic Press, New York, 1976.

3. M. Davis, Applied non standard analysis, Wiley, New York, 1977.

4. (a) P. J. Kelemen and A. Robinson, The non standard $\lambda$ : $\phi_{2}^{4}(x)$ : model: $I$. The technique of non standard analysis in theoretical physics, J. Mathematical Phys. 13 (1972), 1870-1874; II. The standard model from a non standard point of view, J. Mathematical Phys. 13 (1972), 1875-1878.

(b) P. Blandhard and J. Tarski, Renormalizable interactions in two dimensions and sharp-time fields, ZIF, Universität Bielefeld Preprint, 1976.

5. F. A. Berezin and L. D. Faddeev, A remark on Schrödinger's equation with a singular potential, Soviet Math. Dokl. 2 (1961), 372-375.

6. (a) S. Albeverio and R. Høegh-Krohn, Quasi-invariant measures, symmetric diffusion processes and quantum fields, in Les Méthodes Mathématiques de la Théorie Quantique des Champs, Colloques Internat. du CNRS, no. 248, C.N.R.S. 1976, pp. 11-59.

(b) S. Albeverio, R. Hбegh-Krohn and L. Streit, Energy forms, Hamiltonians and distorted Brownian paths, J. Mathematical Phys. 18 (1977), 907-917.

7. (a) C. N. Friedman, Perturbations of the Schrödinger equation by potentials with small support; semi-group product formulas, and applications to quantum mechanics, Ph.D. Dissertation, Princeton University, 1971.

(b) _ Perturbations of the Schrödinger equation by potentials with small support, J. Functional Analysis 10 (1972), 346-360.

8. T. P. Schonbek, Notes on a paper by C. N. Friedman, J. Functional Analysis 14 (1973), 281-294.

9. E. Nelson, Internal set theory: a new approach to non standard analysis. (Expanded version of an invited address given at the Summer 1976 Meeting of the AMS in Toronto.) 
10. M. Reed and B. Simon, Methods of modern mathematical physics. II. Fourier analysis, self-adjointness, Academic Press, New York, 1975.

Mathibatisk Institutt, Universitetet I Oslo, Blindern, Oslo 3, Norway (Current address of J. E. Fenstad and R. Hoegh-Krohn)

Centre de Physique Theorique, CNRS Marseille, F-13274 Marsemlle Cedex, France

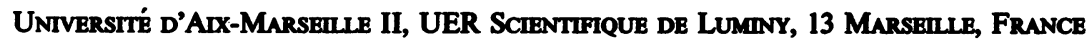

Fakultät für Mathimatik, Universträt Biblbfbid, D4800 Biblbifidd 1, Fedbral Rbpubuc of Germany (Current adderss of S. Albeverio) 